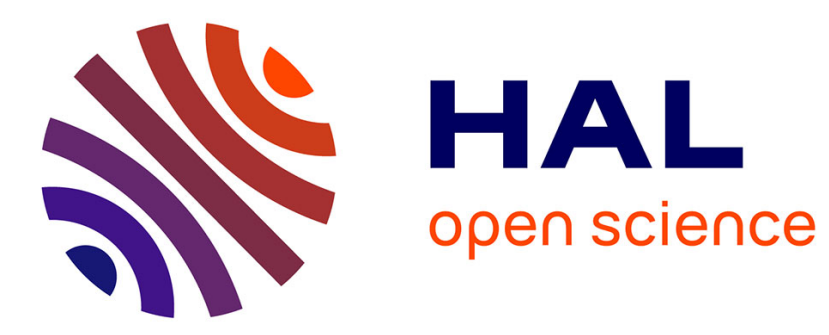

\title{
Polyhedra Associated with Open Locating-Dominating and Locating Total-Dominating Sets in Graphs
}

Gabriela Argiroffo, Silvia Bianchi, Yanina Lucarini, Annegret K Wagler

\section{To cite this version:}

Gabriela Argiroffo, Silvia Bianchi, Yanina Lucarini, Annegret K Wagler. Polyhedra Associated with Open Locating-Dominating and Locating Total-Dominating Sets in Graphs. Combinatorial Optimization. ISCO 2020, pp.3-14, 2020, Print 978-3-030-53261-1 / Online 978-3-030-53262-8. 10.1007/978-3030-53262-8_1. hal-03152094

\section{HAL Id: hal-03152094 \\ https://hal.uca.fr/hal-03152094}

Submitted on 25 Feb 2021

HAL is a multi-disciplinary open access archive for the deposit and dissemination of scientific research documents, whether they are published or not. The documents may come from teaching and research institutions in France or abroad, or from public or private research centers.
L'archive ouverte pluridisciplinaire HAL, est destinée au dépôt et à la diffusion de documents scientifiques de niveau recherche, publiés ou non, émanant des établissements d'enseignement et de recherche français ou étrangers, des laboratoires publics ou privés. 


\title{
Polyhedra associated with open locating-dominating and locating total-dominating sets in graphs
}

\author{
G. Argiroffo, S. Bianchi, Y. Lucarini \\ Universidad Nacional de Rosario, Rosario, Argentina \\ \{garua,sbianchi,lucarini\}fceia.unr.edu.ar \\ Annegret Wagler \\ LIMOS (UMR 6158 CNRS), Université Clermont Auvergne, Clermont-Ferrand, France \\ annegret.wagler@uca.fr
}

\begin{abstract}
The problems of determining open locating-dominating or locating total-dominating sets of minimum cardinality in a graph $G$ are variations of the classical minimum dominating set problem in $G$ and are all known to be hard for general graphs. A typical line of attack is therefore to determine the cardinality of minimum such sets in special graphs.

In this work we study the two problems from a polyhedral point of view. We provide the according linear relaxations, discuss their combinatorial structure, and demonstrate how the associated polyhedra can be entirely described or polyhedral arguments can be applied to find minimum such sets for special graphs.
\end{abstract}

Keywords: open locating-dominating code problem, locating total-dominating code problem, polyhedral approach

\section{Introduction}

For a graph $G$ that models a facility, detection devices can be placed at its nodes to locate an intruder (like a fire, a thief or a saboteur). Depending on the features of the detection devices (to detect an intruder only if it is present at the node where the detector is installed and/or also at any of its neighbors), different dominating sets can be used to determine the optimal distribution of the detection devices in $G$. In the following, we study three problems arising in this context which all have been actively studied during the last decade, see e.g. the bibliography maintained by Lobstein [16].

Let $G=(V, E)$ be a graph. The open neighborhood of a node $i$ is the set $N(i)$ of all nodes of $G$ adjacent to $i$, and $N[i]=\{i\} \cup N(i)$ is the closed 
neighborhood of $i$. A subset $C \subseteq V$ is dominating (resp. total-dominating) if $N[i] \cap C$ (resp. $N(i) \cap C$ ) are non-empty sets for all $i \in V$. A subset $C \subseteq V$ is:

- an identifying code (ID) if it is a dominating set and $N[i] \cap C \neq N[j] \cap C$, for distinct $i, j \in V[15]$;

- an open locating-dominating set (OLD) if it is a total-dominating set and $N(i) \cap C \neq N(j) \cap C$, for distinct $i, j \in V[20]$;

- a locating total-dominating set (LTD) if it is a total-dominating set and $N(i) \cap C \neq N(j) \cap C$, for distinct $i, j \in V-C$ [13].

Figure 1 illustrates the three concepts.

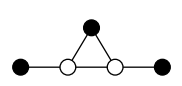

(a)

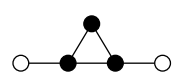

(b)

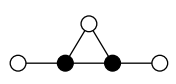

(c)

Figure 1: A graph where the black nodes form a minimum (a) ID-code, (b) OLD-set, (c) LTD-set.

Note that a graph $G$ admits an $I D$-code (or is identifiable) only if there are no true twins in $G$, i.e., there is no pair of distinct nodes $i, j \in V$ such that $N[i]=N[j]$, see [15]. Analogously, a graph $G$ without isolated nodes admits an $O L D$-set if there are no false twins in $G$, i.e., there is no pair of distinct nodes $i, j \in V$ such that $N(i)=N(j)$, see [20].

Given a graph $G$, for $X \in\{I D, O L D, L T D\}$, the $X$-problem on $G$ is the problem of finding an $X$-set of minimum size of $G$. The size of such a set is called the $X$-number of $G$ and is denoted by $\gamma_{X}(G)$. From the definitions, the following relations hold for any graph $G$ (admitting an $X$-set):

$$
\gamma_{L T D}(G) \leq \gamma_{O L D}(G)
$$

whereas $\gamma_{I D}(G)$ and $\gamma_{O L D}(G)$ are not comparable in general.

Determining $\gamma_{I D}(G)$ is in general NP-hard [9] and even remains hard for several graph classes where other in general hard problems are easy to solve, including bipartite graphs [9], split graphs and interval graphs [10].

Also determining $\gamma_{O L D}(G)$ is in general NP-hard [20] and remains NP-hard for perfect elimination bipartite graphs and APX-complete for chordal graphs with maximum degree 4 [18]. Concerning the $L T D$-problem we observe that it is as hard as the $O L D$-problem by just using the same arguments as in [20].

Typical lines of attack are to determine minimum $I D$-codes of special graphs or to provide bounds for their size. Closed formulas for the exact value of $\gamma_{I D}(G)$ have been found so far only for restricted graph families (e.g. for paths and cycles by [8], for stars by [12], and for multipartite graphs, some suns and split graphs by $[2,3,4,5])$. Closed formulas for the exact value of $\gamma_{O L D}(G)$ have been found so far only for cliques and paths [20], some algorithmic aspects are discussed in [18]. Bounds for the $L T D$-number of trees are given in [13, 14], 
whereas the $L T D$-number in special families of graphs, including cubic graphs and grid graphs, is investigated in [14].

As polyhedral methods have been already proved to be successful for several other NP-hard combinatorial optimization problems, it was suggested in [2] to apply such techniques to the $I D$-problem. For that, the following reformulation as set covering problem has been proposed.

For a 0/1-matrix $M$ with $n$ columns, the set covering polyhedron is $Q^{*}(M)=$ $\operatorname{conv}\left\{\mathbf{x} \in \mathbb{Z}_{+}^{n}: M \mathbf{x} \geq \mathbf{1}\right\}$ and $Q(M)=\left\{\mathbf{x} \in \mathbb{R}_{+}^{n}: M \mathbf{x} \geq \mathbf{1}\right\}$ is its linear relaxation. A cover of $M$ is a $0 / 1$-vector $\mathbf{x}$ such that $M \mathbf{x} \geq \mathbf{1}$, and the covering number $\tau(M)$ equals $\min \mathbf{1}^{T} \mathbf{x}, \mathbf{x} \in Q^{*}(M)$.

We obtain such a constraint system $M \mathbf{x} \geq \mathbf{1}$ for the $I D$-problem as follows. Consider a graph $G=(V, E)$. Domination clearly requires that any $I D$-code $C$ intersects the closed neighborhood $N[i]$ of each node $i \in V$; separation means that no two intersections $C \cap N[i]$ and $C \cap N[j]$ are equal. The latter condition can be reformulated that $C$ intersects each symmetric difference $N[i] \triangle N[j]$ for distinct nodes $i, j \in V$. It was shown in [2] that only symmetric differences matter if the nodes $i, j \in V$ have distance $\operatorname{dist}(i, j)=1$ (i.e., are adjacent) or distance $\operatorname{dist}(i, j)=2$ (i.e., are non-adjacent but have a common neighbor).

Hence, determining a minimum $I D$-code in a graph $G=(V, E)$ can be formulated as set covering problem $\min \mathbf{1}^{T} \mathbf{x}, M_{I D}(G) \mathbf{x} \geq \mathbf{1}, \mathbf{x} \in\{0,1\}^{|V|}$ by:

$$
\begin{array}{rll}
\min \mathbf{1}^{T} \mathbf{x} & & \\
x(N[j])=\sum_{i \in N[j]} x_{i} & \geq 1 \forall j \in V & \text { (domination) } \\
x(N[j] \triangle N[k])=\sum_{i \in N[j] \triangle N[k]} x_{i} & \geq 1 \forall j, k \in V, j \neq k & \text { (separation) } \\
\mathbf{x} & \in\{0,1\}^{|V|} &
\end{array}
$$

By [2], the matrix $M_{I D}(G)$ encoding row-wise the closed neighborhoods of the nodes and their symmetric differences is called the identifying code matrix of $G$, and the identifying code polyhedron of $G$ is defined as

$$
P_{I D}(G)=Q^{*}\left(M_{I D}\right)=\operatorname{conv}\left\{\mathbf{x} \in \mathbb{Z}_{+}^{|V|}: M_{I D}(G) \mathbf{x} \geq \mathbf{1}\right\} .
$$

It is clear by construction that a graph is identifiable if and only if none of the symmetric differences results in a zero-row of $M_{I D}(G)$, and that $\gamma_{I D}(G)$ equals the covering number $\tau\left(M_{I D}(G)\right)$.

It turned out that studying the $I D$-problem from a polyhedral point of view can lead to interesting results, see e.g. $[2,3,4,5]$. The aim of this paper is to apply the polyhedral approach to find minimum $O L D$ - or $L T D$-sets.

In Section 2, we give the according definitions of the matrices $M_{O L D}(G)$ and $M_{L T D}(G)$ and of the associated polyhedra, provide some basic properties of the polyhedra $P_{O L D}(G)$ and $P_{L T D}(G)$ and introduce their canonical linear relaxations. Afterwards, we discuss several lines to apply polyhedral techniques.

In Section 3, we present cases where $M_{O L D}(G)$ or $M_{L T D}(G)$ are composed of matrices for which the set covering polyhedron is known and we, thus, immediately can obtain a complete description of $P_{O L D}(G)$ or $P_{L T D}(G)$ and the exact value of $\gamma_{O L D}(G)$ or $\gamma_{L T D}(G)$. 
This demonstrates how polyhedral techniques can be applied in this context. We close with a discussion on future lines of research, including how the here obtained results can be extended to other classes of graphs.

\section{Polyhedra associated to $O L D$ - and $L T D$-sets}

In order to apply the polyhedral approach to the $O L D$ - and the $L T D$-problem, we first give according reformulations as set covering problem.

Theorem 1 Let $G=(V, E)$ be a graph.

(a) Let $G$ have neither isolated nodes nor false twins. $C \subseteq V$ is an OLD-set if and only if $C$ has a non-empty intersection with

$O L D_{1} N(i)$ for all $i \in V$,

$O L D_{2} N(i) \triangle N(j)$ for all distinct $i, j \in V$ with $\operatorname{dist}(i, j) \in\{1,2\}$;

(b) $C \subseteq V$ is an LTD-set if and only if $C$ has a non-empty intersection with

$L T D_{1} \quad N(i)$ for all $i \in V$,

$L T D_{2} N(i) \triangle N(j)$ for all distinct $i, j \in V$ with $\operatorname{dist}(i, j)=1$,

$L T D_{3} \quad N[i] \triangle N[j]$ for all distinct $i, j \in V$ with $\operatorname{dist}(i, j)=2$.

The matrices $M_{O L D}(G)$ and $M_{L T D}(G)$ encoding row-wise the open neighborhoods and their respective symmetric differences read, therefore, as

$$
M_{O L D}(G)=\left(\begin{array}{c}
N(G) \\
\triangle_{1}(i, j) \\
\triangle_{2}(i, j)
\end{array}\right) \quad M_{L T D}(G)=\left(\begin{array}{c}
N(G) \\
\triangle_{1}(i, j) \\
\triangle_{2}[i, j]
\end{array}\right)
$$

where every row in $N(G)$ is the characteristic vector of an open neighborhood of a node in $G$ and $\triangle_{k}(i, j)$ (resp. $\left.\triangle_{k}[i, j]\right)$ is composed of the characteristic vectors of the symmetric difference of open (resp. closed) neighborhoods of nodes at distance $k$. We define by

$$
P_{X}(G)=Q^{*}\left(M_{X}(G)\right)=\operatorname{conv}\left\{\mathbf{x} \in \mathbb{Z}_{+}^{|V|}: M_{X}(G) \mathbf{x} \geq \mathbf{1}\right\}
$$

the $X$-polyhedron for $X \in\{O L D, L T D\}$. We first address the dimension of the two polyhedra. It is known from Balas and $\mathrm{Ng}[7]$ that a set covering polyhedron $Q^{*}(M)$ is full-dimensional if and only if the matrix $M$ has at least two ones per row.

From the submatrix $N(G)$ encoding the open neighborhoods, we see that

$$
V_{N}(G)=\{k \in V:\{k\}=N(i), i \in V\}
$$

are the cases that result in a row with only one 1-entry. From the submatrix $\triangle_{1}(i, j)$, every row has at least two 1-entries (namely for $i$ and $j$ ). From the submatrix $\triangle_{2}(i, j)$, we see that

$$
V_{2}(G)=\{k \in V(G):\{k\}=N(i) \triangle N(j), i, j \in V\}
$$


are the cases that result in a row with only one 1-entry, whereas every row from the submatrix $\triangle_{2}[i, j]$ has at least two 1-entries (namely for $i$ and $j$ ).

We conclude:

Corollary 2 Let $G=(V, E)$ be a graph. We have

- $\operatorname{dim}\left(P_{O L D}(G)\right)=\left|V-V_{N}(G)-V_{2}(G)\right|$ and the constraint $x_{i} \geq 0$ defines a facet of $P_{O L D}(G)$ if and only if $i \notin V_{N}(G) \cup V_{2}(G)$, provided that $G$ has neither isolated nodes nor false twins;

- $\operatorname{dim}\left(P_{L T D}(G)\right)=\left|V-V_{N}(G)\right|$ and the constraint $x_{i} \geq 0$ defines a facet of $P_{O L D}(G)$ if and only if $i \notin V_{N}(G)$.

In addition, $M_{O L D}(G)$ and $M_{L T D}(G)$ may contain redundant rows, where we say that $\mathbf{y}$ is redundant if $\mathbf{x}$ and $\mathbf{y}$ are two rows of $M$ and $\mathbf{x} \leq \mathbf{y}$. As the covering number of a matrix does not change after removing redundant rows, we define the corresponding clutter matrices $C_{O L D}(G)$ and $C_{L T D}(G)$, obtained by removing redundant rows from $M_{O L D}(G)$ and $M_{L T D}(G)$, respectively. We clearly have

$$
P_{X}(G)=Q^{*}\left(C_{X}(G)\right)=\operatorname{conv}\left\{\mathbf{x} \in \mathbb{Z}_{+}^{|V|}: C_{X}(G) \mathbf{x} \geq \mathbf{1}\right\}
$$

for $X \in\{O L D, L T D\}$. Moreover, also in [7] it is proved that the only facetdefining inequalities of a set covering polyhedron $Q^{*}(M)$ with integer coefficients and right hand side equal to 1 are those of the system $M \mathbf{x} \geq \mathbf{1}$. Hence we have:

Corollary 3 All constraints from $C_{X}(G) \mathbf{x} \geq \mathbf{1}$ define facets of $P_{X D}(G)$ for $X \in\{O L D, L T D\}$.

We obtain the corresponding linear relaxations, the fractional OLD-polyhedron $Q_{O L D}(G)$ and the fractional LTD-polyhedron $Q_{L T D}(G)$ of $G$, by considering all vectors satisfying the above inequalities:

$$
Q_{X}(G)=Q\left(C_{X}(G)\right)=\left\{\mathbf{x} \in \mathbb{R}_{+}^{|V|}: C_{X}(G) \mathbf{x} \geq \mathbf{1}\right\}
$$

for $X \in\{O L D, L T D\}$. To study the two problems from a polyhedral point of view, we propose to firstly determine the clutter matrices $C_{O L D}(G)$ and $C_{L T D}(G)$ and then to determine which further constraints have to be added to $Q_{O L D}(G)$ and $Q_{L T D}(G)$ in order to obtain $P_{O L D}(G)$ and $P_{L T D}(G)$, respectively.

\section{$3 \quad$ Complete $p$-partite graphs}

In this section, we consider complete $p$-partite graphs and establish a connection to so-called complete 2-roses of order $n$. Given $n>q \geq 2$, let $\mathcal{R}_{n}^{q}=(V, \mathcal{E})$ be the hypergraph where $V=\{1, \ldots, n\}$ and $\mathcal{E}$ contains all $q$-element subsets of $V$. Nobili and Sassano [17] called the incidence matrix of $\mathcal{R}_{n}^{q}$ the complete q-rose of order $n$ and we denote it by $M\left(\mathcal{R}_{n}^{q}\right)$. In [6], it was shown: 
Theorem $4([6,2])$ The covering polyhedron $Q^{*}\left(M\left(\mathcal{R}_{n}^{q}\right)\right)$ is given by

$$
x\left(V^{\prime}\right) \geq\left|V^{\prime}\right|-q+1
$$

for all subsets $V^{\prime} \subseteq\{1, \ldots, n\}$ with $\left|V^{\prime}\right| \geq q$.

Note that the above inequalities yield, for $\left|V^{\prime}\right|=1$ and $q=2$, the nonnegativity constraints $x_{i} \geq 0$ for all $i \in\{1, \ldots, n\}$.

Complete bipartite graphs First we consider complete bipartite graphs $K_{m, n}$ with bipartition $A=\{1, \ldots, m\}$ and $B=\{m+1, \ldots, m+n\}$. We note that $K_{m, n}$ has false twins (unless $m=1=n$ ) and, thus, no $O L D$-set, hence we only analyse $L T D$-sets. We begin with the case of stars $K_{1, n}$, i.e., $A=\{1\}$ and $n \geq 2$. Note that $K_{1,2}=P_{3}$ and it is easy to see that $\gamma_{L T D}\left(K_{1,2}\right)=2$ holds.

Lemma 5 For a star $K_{1, n}$ with $n \geq 3$, we have

$$
C_{L T D}\left(K_{1, n}\right)=\left(\begin{array}{c|ccc}
1 & 0 & \ldots & 0 \\
\hline 0 & & & \\
\vdots & & M\left(\mathcal{R}_{n}^{2}\right) & \\
0 & & &
\end{array}\right) .
$$

From the above description of the facets of the covering polyhedron associated with complete $q$-roses by [2], we conclude:

Corollary $6 P_{L T D}\left(K_{1, n}\right)$ with $n \geq 3$ is described by the inequalities $x_{1} \geq 1$ and $x\left(B^{\prime}\right) \geq\left|B^{\prime}\right|-1$ for all nonempty subsets $B^{\prime} \subseteq\{2, \ldots, n+1\}$.

Furthermore, combining $x_{1} \geq 1$ and $x(B) \geq|B|-1$ yields the full rank constraint $x(V) \geq|B|$ which immediately implies $\gamma_{L T D}\left(K_{1, n}\right)=|V|-1=n$ (and provides an alternative proof for the result given in [14]).

Observe that for $K_{2,2}$, it is easy to see that $\gamma_{L T D}\left(K_{2,2}\right)=2$. For general complete bipartite graphs $K_{m, n}$ with $m \geq 2, n \geq 3$, we obtain:

Lemma 7 For a complete bipartite graph $K_{m, n}$ with $m \geq 2, n \geq 3$, we have

$$
C_{L T D}\left(K_{m, n}\right)=\left(\begin{array}{cc}
M\left(\mathcal{R}_{m}^{2}\right) & 0 \\
0 & M\left(\mathcal{R}_{n}^{2}\right)
\end{array}\right) .
$$

Note that results from [2] show that $C_{I D}\left(K_{m, n}\right)=C_{L T D}\left(K_{m, n}\right)$. Hence, we directly conclude from the facet description of $P_{I D}\left(K_{m, n}\right)$ by [2]:

Corollary $8 P_{L T D}\left(K_{m, n}\right)$ is given by the inequalities

1. $x(C) \geq|C|-1$ for all nonempty $C \subseteq A$,

2. $x(C) \geq|C|-1$ for all nonempty $C \subseteq B$.

Moreover, $\gamma_{L T D}\left(K_{m, n}\right)=|V|-2=m+n-2$.

This provides an alternative proof for the result given in [14]. 
Complete $p$-partite graphs The results above can be further generalized for complete $p$-partite graphs. Consider $K_{n_{1}, \ldots, n_{p}}=\left(U_{1} \cup \cdots \cup U_{p}, E\right)$ where each $U_{i}$ induces a nonempty stable set and all edges between $U_{i}$ and $U_{j}, i \neq j$ are present. We use $\left|U_{i}\right|=n_{i}$ for $i=1, \ldots, p,|V|=n$ and assume $n_{1} \leq n_{2} \leq$ $\ldots \leq n_{p}$ as well as $p \geq 3$. For illustration, complete 3-partite and 4-partite graphs are depicted in Figure 2.

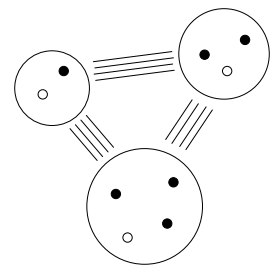

(a)

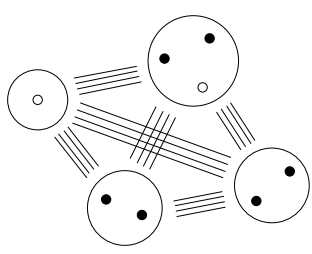

(b)

Figure 2: (a) A complete 3-partite graph with $n_{1}=2, n_{2}=3$ and $n_{3}=4$, (b) A complete 4-partite graph with $n_{1}=1, n_{2}=n_{3}=2$ and $n_{4}=3$.

We note that $K_{n_{1}, \ldots, n_{p}}$ has false twins and, thus, no $O L D$-set, unless $n_{1}=$ $\cdots=n_{p}=1$ and the graph is a clique.

Lemma 9 Let $K_{n_{1}, n_{2}, \ldots, n_{p}}$ be a complete p-partite graph.

(a) If $n_{1}=\cdots=n_{p}=1$, then $K_{n_{1}, n_{2}, \ldots, n_{p}}$ equals the clique $K_{p}$ and

$$
C_{O L D}\left(K_{n_{1}, n_{2}, \ldots, n_{p}}\right)=C_{L T D}\left(K_{n_{1}, n_{2}, \ldots, n_{p}}\right)=M\left(\mathcal{R}_{p}^{2}\right) .
$$

(b) If $n_{1}=\cdots=n_{r}=1$ with $r \geq 2$ and $n_{r+1} \geq 2$, then

$$
C_{L T D}\left(K_{n_{1}, n_{2}, \ldots, n_{p}}\right)=\left(\begin{array}{ccccc}
M\left(\mathcal{R}_{r}^{2}\right) & 0 & 0 & \ldots & 0 \\
0 & M\left(\mathcal{R}_{n_{r+1}}^{2}\right) & 0 & \cdots & 0 \\
\vdots & & \ddots & & \vdots \\
0 & & \cdots & & M\left(\mathcal{R}_{n_{p}}^{2}\right)
\end{array}\right) .
$$

(c) If $n_{1}=1$ and $n_{2} \geq 2$, then

$$
C_{L T D}\left(K_{n_{1}, n_{2}, \ldots, n_{p}}\right)=\left(\begin{array}{cccccc}
0 & M\left(\mathcal{R}_{n_{2}}^{2}\right) & 0 & 0 & \ldots & 0 \\
0 & 0 & M\left(\mathcal{R}_{n_{3}}^{2}\right) & 0 & \ldots & 0 \\
\vdots & & \ddots & \ddots & & \vdots \\
0 & 0 & \ldots & & & M\left(\mathcal{R}_{n_{p}}^{2}\right)
\end{array}\right) .
$$

(d) If $n_{1} \geq 2$, then

$$
C_{L T D}\left(K_{n_{1}, n_{2}, \ldots, n_{p}}\right)=\left(\begin{array}{ccccc}
M\left(\mathcal{R}_{n_{1}}^{2}\right) & 0 & 0 & \ldots & 0 \\
0 & M\left(\mathcal{R}_{n_{2}}^{2}\right) & 0 & \ldots & 0 \\
\vdots & & \ddots & & \vdots \\
0 & & \cdots & & M\left(\mathcal{R}_{n_{p}}^{2}\right)
\end{array}\right) .
$$


From the description of the facets of the covering polyhedron associated with complete $q$-roses by [2] and taking the block structure of the matrices into account, we conclude:

Corollary 10 Let $K_{n_{1}, n_{2}, \ldots, n_{p}}$ be a complete p-partite graph.

(a) If $n_{1}=\cdots=n_{p}=1$, then $P_{X}\left(K_{n_{1}, n_{2}, \ldots, n_{p}}\right)$ is given by the inequalities

- $x\left(V^{\prime}\right) \geq\left|V^{\prime}\right|-1$ for all nonempty subsets $V^{\prime} \subseteq V$

and $\gamma_{X}\left(K_{n_{1}, n_{2}, \ldots, n_{p}}\right)=n-1$ for $X \in\{O L D, L T D\}$.

(b) If $n_{1}=\cdots=n_{r}=1$ with $r \geq 2$ and $n_{r+1} \geq 2$, then

- $x\left(V^{\prime}\right) \geq\left|V^{\prime}\right|-1$ for all nonempty subsets $V^{\prime} \subseteq U_{1} \cup \cdots \cup U_{r}$,

- $x\left(V^{\prime}\right) \geq\left|V^{\prime}\right|-1$ for all nonempty subsets $V^{\prime} \subseteq U_{i}$ for $i \in\{r+1, \ldots, p\}$

and $\gamma_{L T D}\left(K_{n_{1}, n_{2}, \ldots, n_{p}}\right)=n-p+r-1$.

(c) If $n_{1}=1$ and $n_{2} \geq 2$, then $P_{L T D}\left(K_{n_{1}, n_{2}, \ldots, n_{p}}\right)$ is given by the inequalities

- $x\left(V^{\prime}\right) \geq\left|V^{\prime}\right|-1$ for all nonempty subsets $V^{\prime} \subseteq U_{i}$ for $i \in\{2, \ldots, p\}$

and $\gamma_{L T D}\left(K_{n_{1}, n_{2}, \ldots, n_{p}}\right)=n-p$.

(d) If $n_{1} \geq 2$, then $P_{L T D}\left(K_{n_{1}, n_{2}, \ldots, n_{p}}\right)$ is given by the inequalities

- $x\left(V^{\prime}\right) \geq\left|V^{\prime}\right|-1$ for all nonempty subsets $V^{\prime} \subseteq U_{i}$ for $i \in\{1, \ldots, p\}$

and $\gamma_{L T D}\left(K_{n_{1}, n_{2}, \ldots, n_{p}}\right)=n-p$.

Corrollary 10(a) provides an alternative proof for the result on $O L D$-sets in cliques given in [20].

\section{Some families of split graphs}

A graph $G=(C \cup S, E)$ is a split graph if its node set can be partitioned into a clique $C$ and a stable set $S$. Split graphs are closed under taking complements and form the complementary core of chordal graphs since $G$ is a split graph if and only if $G$ and $\bar{G}$ are chordal or if and only if $G$ is $\left(C_{4}, \bar{C}_{4}, C_{5}\right)$-free [11].

Our aim is to study LTD-sets in some families of split graphs having a regular structure from a polyhedral point of view.

Complete split graphs. A complete split graph is a split graph where all edges between $C$ and $S$ are present. Complete split graphs can be seen as special case of complete multi-partite graphs studied in Section 3. In fact, a complete split graph is a clique if $|S|=1$, a star if $|C|=1$, and a crown if $|C|=2$, see Fig. 3(a),(b). Otherwise, the graph can be seen as a complete multi-partite graph where all parts but one have size 1, i.e. as $K_{n_{1}, n_{2}, \ldots, n_{p}}$ with $n_{1}=\cdots=n_{p-1}=1$ and $n_{p} \geq 2$ such that $U_{1} \cup \cdots \cup U_{p-1}$ induce the clique $C$ and $U_{p}$ the stable set $S$. Hence, we directly conclude from Lemma 9 and Corollary 10: 


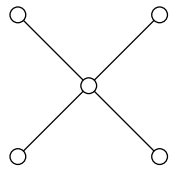

(a)

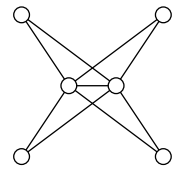

(b)

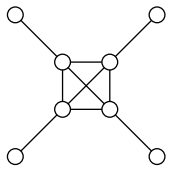

(c)

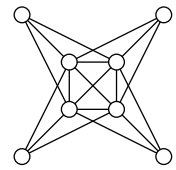

(d)

Figure 3: (a) star, (b) crown, (c) thin headless spider, (d) thick headless spider.

Corollary 11 Let $G=(C \cup S, E)$ be a complete split graph.

(a) If $|S|=1$, then $G$ is a clique,

$$
C_{X}(G)=M\left(\mathcal{R}_{|C|+1}^{2}\right)
$$

and $\gamma_{X}(G)=|C|$ for $X \in\{O L D, L T D\}$.

(b) If $|C|=1$, then $G$ is a star,

$$
C_{L T D}(G)=\left(\begin{array}{c|ccc}
1 & 0 & \ldots & 0 \\
\hline 0 & & & \\
\vdots & & M\left(\mathcal{R}_{|S|}^{2}\right) & \\
0 & & &
\end{array}\right) .
$$

and $\gamma_{L T D}(G)=|S|$.

(c) Otherwise, we have

$$
\begin{aligned}
& C_{L T D}(G)=\left(\begin{array}{cc}
M\left(\mathcal{R}_{|C|}^{2}\right) & 0 \\
0 & M\left(\mathcal{R}_{|S|}^{2}\right)
\end{array}\right) \\
& \text { and } \gamma_{L T D}(G)=|S|+|C|-2 \text {. }
\end{aligned}
$$

Headless spiders. A headless spider is a split graph with $C=\left\{c_{1}, \ldots, c_{k}\right\}$ and $S=\left\{s_{1}, \ldots, s_{k}\right\}$; it is thin (resp. thick) if $s_{i}$ is adjacent to $c_{j}$ if and only if $i=j$ (resp. $i \neq j$ ), see Figure 3(c),(d) for illustration. Clearly, the complement of a thin spider is a thick spider, and vice-versa. It is easy to see that for $k=2$, the path $P_{4}$ equals the thin and thick headless spider. Moreover, it is easy to check that headless spiders are twin-free.

A thick headless spider with $k=3$ equals the 3 -sun $S_{3}$ and it is easy to see that $\gamma_{O L D}\left(S_{3}\right)=4$ and $\gamma_{L T D}\left(S_{3}\right)=3$ holds. To describe the clutters for $k \geq 4$, we use the following notations. Let $J_{n}$ denote the $n \times n$ matrix having 1-entries only and $I_{n}$ the $n \times n$ identity matrix. Furthermore, let $J_{n-1, n}(i)$ denote a matrix s.t. its $i$-th column has 0 -entries only and removing the $i$-th column results in $J_{n-1}$, and $I_{n-1, n}(j)$ denote a matrix s.t. its $j$-th column has 1-entries only and removing the $j$-th column results in $I_{n-1}$. 
Lemma 12 For a thick headless spider $G=(C \cup S, E)$ with $k \geq 4$, we have

$$
C_{O L D}(G)=\left(\begin{array}{cc}
M\left(\mathcal{R}_{|S|}^{|S|-1}\right) & 0 \\
0 & M\left(\mathcal{R}_{|C|}^{2}\right)
\end{array}\right)
$$

and

$$
C_{L T D}(G)=\left(\begin{array}{cc}
0 & M\left(\mathcal{R}_{|C|}^{|C|-1}\right) \\
J_{k-1, k}(k) & I_{k-1, k}(k) \\
\vdots & \vdots \\
J_{k-1, k}(1) & I_{k-1, k}(1) \\
M\left(\mathcal{R}_{|S|}^{2}\right) & M\left(\mathcal{R}_{|C|}^{2}\right) \\
J_{|S|} & I_{|C|}
\end{array}\right)
$$

From the description of the polyhedron associated with complete $q$-roses by [2] and taking the block structure of $C_{O L D}(G)$ into account, we conclude:

Corollary 13 For a thick headless spider $G=(C \cup S, E)$ with $k \geq 4, P_{O L D}(G)$ is given by the inequalities

- $x_{i} \geq 0$ for all $i \in C \cup S$,

- $x\left(S^{\prime}\right) \geq\left|S^{\prime}\right|-k+2$ for all $S^{\prime} \subseteq S$ with $\left|S^{\prime}\right| \geq k-1$,

- $x\left(C^{\prime}\right) \geq\left|C^{\prime}\right|-1$ for all $C^{\prime} \subseteq C$ with $\left|C^{\prime}\right| \geq 2$,

and $\gamma_{O L D}(G)=|C|+1$.

On the other hand, from the clutter matrix $C_{L T D}(G)$, we immediately see that $C$ is an $L T D$-set. However, $C$ is a minimum $L T D$-set only if $k=4$. For thick headless spiders with $k \geq 5$, we can show, using polyhedral arguments, that $k-1$ is a lower bound for the cardinality of any $L T D$-set. Exhibiting an $L T D$-set of size $k-1$ thus ensures minimality:

Theorem 14 For a thick headless spider $G=(C \cup S, E)$ with $k \geq 5$, we have $\gamma_{L T D}(G)=k-1$.

The situation is different for thin headless spiders:

Lemma 15 For a thin headless spider $G=(C \cup S, E)$ with $k \geq 3$, we have

$$
C_{O L D}(G)=C_{L T D}(G)=\left(\begin{array}{ll}
0 & I_{|C|}
\end{array}\right) .
$$

We immediately conclude:

Corollary 16 For a thin headless spider $G=(C \cup S, E)$ with $k \geq 3, P_{X}(G)$ is given by the inequalities

- $x_{i} \geq 1$ for all $i \in C$,

$C$ is the unique $X$-set and $\gamma_{X}(G)=|C|$ follows for $X \in\{O L D, L T D\}$. 


\section{Concluding remarks}

In this paper, we proposed to study the $O L D$ - and $L T D$-problem from a polyhedral point of view, motivated by promising polyhedral results for the $I D$ problem $[2,3,4,5]$. That way, we were able to provide closed formulas for the $L T D$-numbers of all kinds of complete $p$-partite graphs (Section 3), and for the studied families of split graphs as well as the $O L D$-numbers of thin and thick headless spiders (Section 4).

In particular, if we have the same clutter matrix for two different $X$-problems, then we can conclude that every solution of one problem is also a solution for the other problem, and vice versa, such that the two $X$-polyhedra coincide and the two $X$-numbers are equal. This turned out to be the case for

- complete bipartite graphs as $C_{I D}\left(K_{m, n}\right)=C_{L T D}\left(K_{m, n}\right)$ holds by Lemma 7 and results from [2],

- thin headless spiders $G$ as $C_{O L D}(G)=C_{L T D}(G)$ holds by Lemma 15 .

Furthermore, we were able to provide the complete facet descriptions of

- the $L T D$-polyhedra for all complete $p$-partite graphs (including complete split graphs) and for thin headless spiders (see Section 3 and Lemma 15),

- the $O L D$-polyhedra of cliques, thin and thick headless spiders (see Corollary 10 and Section 4).

The complete descriptions of some $X$-polyhedra also provide us with information about the relation between $Q^{*}\left(C_{X}(G)\right)$ and its linear relaxation $Q\left(C_{X}(G)\right)$. A matrix $M$ is ideal if $Q^{*}(M)=Q(M)$. For any nonideal matrix, we can evaluate how far $M$ is from being ideal by considering the inequalties that have to be added to $Q(M)$ in order to obtain $Q^{*}(M)$. With this purpose, in [1], a matrix $M$ is called rank-ideal if only 0/1-valued constraints have to be added to $Q(M)$ to obtain $Q^{*}(M)$. From the complete descriptions obtained in Section 3 and Section 4, we conclude:

Corollary 17 The LTD-clutters and OLD-clutters of thin headless spiders are ideal for all $k \geq 3$.

Corollary 18 The LTD-clutters of all complete p-partite graphs and the OLDclutters of cliques and thick headless spiders are rank-ideal.

Finally, the $L T D$-clutters of thick headless spiders have a more complex structure such that also a facet description of the $L T D$-polyhedra is more involved. However, using polyhedral arguments, is was possible to establish that $k-1$ is a lower bound for the cardinality of any $L T D$-set. Exhibiting an $L T D$ set of size $k-1$ thus allowed us to deduce the exact value of the $L T D$-number of thick headless spiders (Theorem 14).

This demonstrates how the polyhedral approach can be applied to find $X$ sets of minimum size for special graphs $G$, by determining and analyzing the 
$X$-clutters $C_{X}(G)$, even in cases where no complete description of $P_{X}(G)$ is known yet.

As future lines of research, we plan to work on a complete description of the $L T D$-polyhedra of thick headless spiders and to apply similar and more advanced techniques for other graphs in order to obtain either $X$-sets of minimum size or strong lower bounds stemming from linear relaxations of the $X$ polyhedra, enhanced by suitable cutting planes.

\section{References}

[1] Argiroffo, G., Bianchi, S.: On the set covering polyhedron of circulant matrices, Discrete Optimization 6(2), 162-173 (2009)

[2] G. Argiroffo, S. Bianchi, Y. Lucarini, A. Wagler, Polyhedra associated with identifying codes in graphs, Discrete Applied Mathematics 245 (2018) 16-27.

[3] G. Argiroffo, S. Bianchi, A. Wagler: Study of identifying code polyhedra for some families of split graphs, Lecture Notes in Computer Science 8596 (2014) 13-25.

[4] G. Argiroffo, S. Bianchi, A. Wagler: On identifying code polyhedra of families of suns, VIII ALIO/EURO Workshop on Applied Combinatorial Optimization 2014 .

[5] G. Argiroffo, S. Bianchi, A. Wagler: Progress on the description of identifying code polyhedra for some families of split graphs, Discrete Optimization 22 (2016) $225-240$

[6] Argiroffo, G., Carr, M.: On the set covering polyhedron of $q$-roses, In: Proc. of the VI ALIO/EURO Workshop on Applied Combinatorial Optimization 2008, Buenos Aires, Argentina (2008).

[7] E. Balas, S. M. Ng, On the set covering polytope: I. All the facets with coefficients in $\{0,1,2\}$, Mathematical Programming 43, (1989) 57-69.

[8] N. Bertrand, I. Charon, O. Hudry, A. Lobstein, Identifying and locating dominating codes on chains and cycles, Eur. J. Comb. 25 (2004) 969-987.

[9] Charon, I., Hudry, O., Lobstein, A.: Minimizing the size of an identifying or locating-dominating code in a graph is NP-hard, Theoretical Computer Science 290, 2109-2120 (2003)

[10] Foucaud, F.: The complexity of the identifying code problem in restricted graph classes, In: Proc. of the International Workshop on Combinatorial Algorithms (IWOCA 2013), LNCS, vol. 8288, pp. 150-163. Springer, Heidelberg (2013)

[11] Földes, S., Hammer, P.: Split graphs, In: Proc. of the VIII Southeastern Conference on Combinatorics, Graph Theory and Computing (Baton Rouge, La.), Congressus Numerantium XIX, Winnipeg: Utilitas Math., pp. 311-315, (1977)

[12] S. Gravier, J. Moncel, On graphs having a $V\{x\}$-set as an identifying code, Discrete Mathematics 307 (2007) 432-434. 
[13] T.W. Haynes, M. A. Henning, J. Howard, Locating and total-dominating sets in trees, Discrete Applied Mathematics 154, (2006), 1293-1300.

[14] M. A. Henning, N. J. Rad, Locating-total domination in graphs, Discrete Applied Mathematics, 160 (2012) 1986-1993.

[15] Karpovsky, M.G., Chakrabarty, K., Levitin, L.B.: On a new class of codes for identifying vertices in graphs, IEEE Transactions on Information Theory 44, 599611 (1998)

[16] A. Lobstein, Watching systems, identifying, locating-dominating and discriminating codes in graphs, https://www.lri.enst.fr/ lobstein/debutBIBidetlocdom.pdf

[17] P. Nobili, A. Sassano, Facets and lifting procedures for the set covering polytope, Mathematical Programming 45 111-137 (1989).

[18] A. Pandey, Open Neighborhood Locating-Dominating Set in Graphs: Complexity and Algorithms, IEEE, 2015 International Conference on Information Technology (ICIT)

[19] Sassano, A.: On the facial structure of the set covering polytope, Mathematical Programming 44, 181-202 (1989)

[20] S.J. Seo, P.J. Slater, Open neighborhood locating dominating sets, Australasian Journal of Combinatorics, 46, (2010), 109-119. 